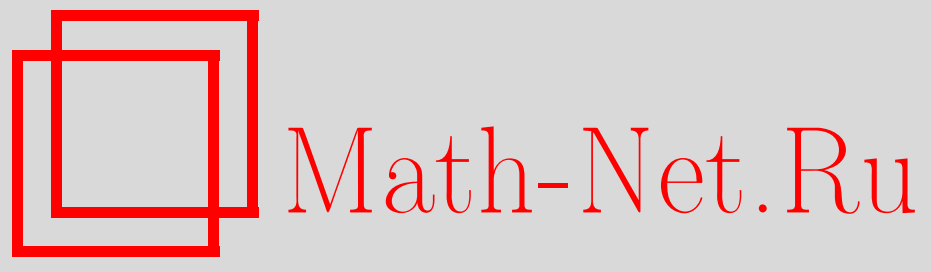

М. В. Бондарко, Канонические представители в классах строгой изоморфности формальных групп, Матем. заметки, 2007, том 82, выпуск 2, 183-189

DOI: https://doi.org/10.4213/mzm3801

Использование Общероссийского математического портала Math-Net.Ru подразумевает, что вы прочитали и согласны с пользовательским соглашением http://www . mathnet.ru/rus/agreement

Параметры загрузки:

IP : 54.92 .164 .108

26 апреля 2023 г., 13:14:39

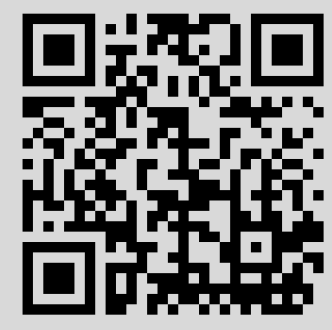


Том 82 выпуск 2 август 2007

УДК 512.741 .5

\section{Канонические представители в классах строгой изоморфности формальных групп}

\section{М. В. Бондарко}

Целью настоящей работы является явное построение канонических представителей в каждом классе строгой изоморфности коммутативных формальных групп над произвольным кольцом без кручения. Отдельно рассматривается случай $\mathbb{Z}_{(p)}$-алгебры. Мы доказываем, что при естественных ограничениях на подкольцо канонические представители формальных групп над ним согласованы с представителями для кольца. Доказаны необходимые и достаточные условия того, что отображение, индуцированное гомоморфизмом колец без кручения на классах строгой изоморфности формальных групп, инъективно и сюръективно.

Библиография: 4 названия.

\section{Введение}

Целью этой работы является построение канонических представителей в классах строгой изоморфности формальных групповых законов. Для произвольного кольца $R$ без кручения и каждого простого $p \in \mathbb{Z}$ мы выбираем систему представителей $\theta_{p}: R / p R \rightarrow R$. Мы доказываем, что любая формальная группа $F$ над $R$ строго изоморфна ровно одной формальной группе $\theta(F)$, чьи коэффициенты при выражении через универсальный криволинейный закон (см. [1]) лежат в соответствую-

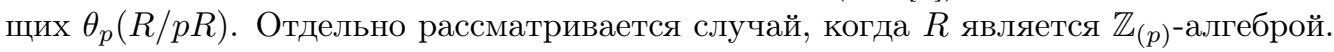
В этом случае выполнен аналогичный результат для универсального $p$-типического закона. Для кольца целых неразветвленного локального поля эти канонические представители классов строгой изоморфности совпадают с построенными в рабо$\operatorname{таx~[2]~и~[3].~}$

Отметим, что единственный известный автору более ранний результат в этом направлении был получен в работе [4] только для одномерных формальных групп над неразветвленным локальным полем.

Мы также доказываем, что если $S \subset R$ и представители в $S$ согласованы с представителями в $R$, то формальная группа $F$ над $R$ строго изоморфна формальной группе, определенной над $S$, тогда и только тогда, когда канонический представитель $F$ определен над $S$.

Работа выполнена при поддержке Российского фонда фундаментальных исследований (грант № 04-01-00082a). 
В конце работы мы доказываем необходимые и достаточные условия того, что отображение, индуцированное гомоморфизмом колец без кручения на классах строгой изоморфности формальных групп, инъективно и сюръективно.

ОБозначения. Обозначим $\mathbb{P}$ - множество простых чисел, $R$ - коммутативное кольцо с единицей без кручения.

Для каждого простого $p$ будем обозначать кольцо целых чисел, локализованное по $p$, через $\mathbb{Z}_{(p)}$.

Обозначим $X=\left(X_{i}\right)=X_{1}, \ldots, X_{m}$ - формальные переменные; $x$ - одна переменная.

Далее, $F$ будет обозначать коммутативный $m$-мерный формальный групповой закон над $R, \lambda=\left(\lambda_{i}(X)\right)$ - логарифм $F$.

Во всей статье $M_{m}(\mathfrak{A})$ будет обозначать кольцо матриц размера $m \times m$ над (возможно, некоммутативным) кольцом $\mathfrak{A}, I_{m}$ обозначает единичную матрицу размера $m \times m$; запись $a=\left(a_{i}\right)$ означает, что $a-$ столбец, состоящий из $a_{i}, 1 \leqslant i \leqslant m$.

\section{1. Определение и свойства универсальных законов}

Этот раздел посвящен напоминанию нужных нам определений и утверждений из книги [1].

1.1. Криволинейные и $p$-типические группы. Так как в этой статье мы будем изучать только коммутативные формальные группы над кольцами без кручения, наши формальные группы будут иметь логарифмы.

Напомним, что набор рядов $l=\left(l_{i}\left(X_{j}\right)\right)$ называется строгим изоморфизмом между $m$-мерными законами $F$ и $G$, если $l_{i} \equiv X_{i} \bmod \operatorname{deg} 2$ и $G(l(X), l(Y))=l(F(X, Y))$. Для формального группового закона над кольцом $R$ набор $\lambda=\left(\lambda_{i}(X)\right)$ рядов над $\mathbb{Q} R$ называется логарифмом $F$, если $\lambda$ задает строгий изоморфизм $F$ и $F_{+}^{m}$; здесь $F_{+}$обозначает аддитивный формальный групповой закон. Нетрудно видеть, что $m$-мерная группа $F$ строго изоморфна $F_{+}^{m}$ тогда и только тогда, когда все коэффициенты $\lambda_{i}(X)$ лежат в $R$ для всех $1 \leqslant i \leqslant m$.

Напомним также, что формальный групповой закон $F$ называется криволинейным, если $\lambda_{i}$ для каждого $i$ можно представить в виде

$$
X_{i}+\sum_{j, k} a_{i j k} X_{j}^{k}
$$

где $a_{i j k} \in \mathbb{Q} R$. Если, кроме того, для некоторого $p \in \mathbb{P}$ все $\lambda_{i}$ имеют вид

$$
X_{i}+\sum_{j, k} b_{i j k} X_{j}^{p^{k}}
$$

то $F$ называется $p$-типическим формальным групповым законом.

Следующее утверждение хорошо известно.

ПрЕДЛОЖЕНИЕ 1.1.1. Пусть логарифм формального группового закона F равен

$$
\lambda=\left(\lambda_{i}\right)=\left(\sum a_{i_{1} \ldots i_{m}}^{i_{1}} X_{1}^{i_{1}} \cdots X_{m}^{i_{m}}\right) .
$$


Тогда выполнены следующие утверждения.

1. Групповой закон F строго изоморфен криволинейному закону с логарифмом, равнымм

$$
\left(\lambda_{i}^{\prime}\right)=\left(\sum a_{i_{1} \ldots i_{m}}^{\prime i} X_{1}^{i_{1}} \cdots X_{m}^{i_{m}}\right)
$$

Здесъ $a_{i_{1} \ldots i_{m}}^{\prime i}=a_{i_{1} \ldots i_{m}}^{i}$, если $i_{s}=0$ для всех $s$, кроме одного; для всех других мультииндексов коэффичиенты $a_{i_{1} \ldots i_{m}}^{i}$ равны нулю.

2. Пусть $R-\mathbb{Z}_{(p)}$-алгебра для некоторого $p \in \mathbb{P}$. Тогда $F$ строго изоморфен формальной группе с логарифмом, равным $\left(\lambda_{i}^{\prime \prime}\right)$. Здесъ

$$
\lambda_{i}^{\prime \prime}=\sum a_{i_{1} \ldots i_{m}}^{\prime \prime i} X_{1}^{i_{1}} \cdots X_{m}^{i_{m}}
$$

где $a_{i_{1} \ldots i_{m}}^{\prime \prime i}=a_{i_{1} \ldots i_{m}}^{i}$, если $i_{s}=0$ для всех $s$, кроме одного; оставшийся $i_{s}-$ степень $р$, все остальные $a_{i_{1} \ldots i_{m}}^{\prime \prime}$ равны нулю.

Утверждение первой части было доказано в [1] (см. пункт 12.3.6), второе - это теорема 16.4.14 той же книги.

1.2. Универсальные групповые законы и его свойства. В этой статье нам придется пользоваться универсальным $m$-мерным криволинейным законом. Существуют различные групповые законы, удовлетворяющие нужным нам свойствам, их описание несколько громоздко. В частности, в [1] были построены групповые законы $F_{R}$ и $H_{R}($ см. $\S \S 12.2,12.3)$, каждый из них может быть взят в качестве $F_{e}$ (см. ниже). Поэтому мы не будем строить универсальные криволинейные законы в этой работе, лишь опишем необходимые нам свойства.

Мы считаем, что универсальный $m$-мерный криволинейный закон $F_{e}$ определен над кольцом $\mathbb{Z}\left[e_{i j k}\right]$, где $k \geqslant 2$, а $1 \leqslant i, j \leqslant m$. Универсальность означает, что для каждого криволинейного $m$-мерного группового закона $F$ над произвольным кольцом $R$ существует единственный набор $f_{i j k} \in R$ такой, что при подстановке $f_{i j k}$ вместо $e_{i j k}$ в $F_{e}$ мы получаем $F$.

Определим функцию $v$ для $n \in \mathbb{Z}$ следующим образом: $v(n)=p$, если $n$ является положительной степенью простого числа $p$ и $v(n)=1$ для всех остальных $n$.

Мы будем считать, что для каждого $t>1$ существует набор многочленов $d_{i j l t}$ над $\mathbb{Q}$ такой, что логарифм универсального $m$-мерного криволинейного закона $\lambda_{e}=\left(\lambda_{e i}\right)$ удовлетворяет равенству

$$
\lambda_{e i} \equiv \sum_{1 \leqslant j \leqslant m, l<t} d_{i l j t}\left(e_{u v r}\right) X_{j}^{l}+\sum_{j} \frac{e_{i j t}}{v(t)} X_{j}^{t} \quad \bmod \operatorname{deg} t+1,
$$

где $i, u, v$ (независимо) пробегают координаты закона $F_{e}, 2 \leqslant r<t, m$ - размерность формальной группы. Формальные законы $F_{R}$ и $H_{R}$, построенные в [1], удовлетворяют этому свойству согласно [1; формула (12.3.3)].

Нам также понадобится универсальный $m$-мерный $p$-типический групповой закон $F_{c}$, который был определен в [1]. Рассмотрим кольцо $\mathfrak{A}=\mathbb{Z}_{(p)}\left[c_{i j k}\right], k>0$, $1 \leqslant i, j \leqslant m$. Определим на нем кольцевой оператор $\sigma$ с помощью равенства $\sigma\left(c_{i j k}\right)=c_{i j k}^{p}$. Рассмотрим кольцо $\mathfrak{A}^{\prime}$, которое равно $\mathfrak{A}[[\Delta]]$ как левый $\mathfrak{A}$-модуль и удовлетворяет равенству $\Delta^{s} a=\sigma^{s}(a) \Delta^{s}$ для каждого $a \in \mathfrak{A}$ и $s>0$. Тогда 
логарифм $\lambda_{c}=\left(\lambda_{c i}\right)$ универсального $p$-типического $m$-мерного закона $F_{c}=F_{c, m}$ получается при обращении матрицы

$$
u_{c}=I_{m}-\sum_{k>0} p^{-1}\left(c_{i j k}\right) \Delta^{k}
$$

в $M_{m}\left(\mathfrak{A}^{\prime}\right)$ и применении результата к $X=\left(X_{1}, \ldots, X_{m}\right)$ по правилу $a \Delta^{s}\left(X_{j}\right)=a X_{j}^{p^{s}}$ для $1 \leqslant j \leqslant m, s>0$.

Универсальность снова означает, что для каждого $p$-типического $m$-мерного группового закона $F$ над кольцом $R$ существует единственный набор $f_{i j k} \in R$ такой, что при подстановке $f_{i j k}$ вместо $c_{i j k}$ в $F_{c}$ мы получаем $F$.

Нетрудно проверить, что для каждого $t>0$ существует набор многочленов $g_{i j l t}$ над $\mathbb{Q}$ такой, что

$$
\lambda_{c i} \equiv \sum_{1 \leqslant j \leqslant m, 0 \leqslant l<t} g_{i l j t}\left(c_{u v r}\right) X_{j}^{p^{l}}+\sum_{j} \frac{c_{i j t}}{p} X_{j}^{p^{t}} \quad \bmod \operatorname{deg} p^{t+1},
$$

где $i, u, v$ независимо пробегают координаты закона $F_{c}, 1 \leqslant r<t$.

Мы будем считать, что $F_{c}$ и $F_{e}$ обозначают соответствующие универсальные законы размерности $m$.

Отметим, что для произвольного кольца $R$ (не обязательно $\mathbb{Z}_{(p)}$-алгебры) закон $F_{c}$ дает при подстановке $f_{i j k} \in R$ формальную группу над $R$.

\section{2. Основные результаты}

Для каждого $p \in \mathbb{P}$ зафиксируем систему представителей $\theta_{p}: R / p R \rightarrow R$. Мы также определим $\theta_{1}: R / R \rightarrow R$; будем считать, что $\theta_{1}(0)=0$.

\section{1. Канонические представители.}

Теорема 2.1.1. 1. Любая формалъная группа $F$ над $R$ строго изоморфна ровно одной криволинейной формальной группе $\theta(F)$ такой, что все значения коэфбиииентов $e_{i j k}$, при подстановке которых в универсальный криволинейный закон $F_{e}$ получается $\theta(F)$, принадлежат $\theta_{p}(R / p R)$ при всех $i>1$, представимых в виде $p^{l}$, $l>0$, для какого-то простого $p$, и равны нулю при других значениях $i$.

2. Пусть $R-\mathbb{Z}_{(p)}$-алгебра. Тогда любая формальная группа $F$ над $R$ строго изоморфна ровно одной $p$-типической формальной группе $\theta(F)$ такой, что все значения коэффичиентов $c_{i j k}$, при подстановке которых в универсалъный р-типический закон $F_{c}$ получается $\theta(F)$, принадлежат $\theta_{p}(R / p R)$.

ДоКАЗАТЕЛЬСтво. 1. Построим логарифм представителя класса изоморфности группы последовательно по степеням $X$. Пусть для некоторого $s>1$ криволинейная группа $F^{\prime}=F_{s}$ строго изоморфна $F$, коэффициенты $f_{i j l}^{\prime}$, при подстановке которых в $F_{e}$ получается $F^{\prime}$, при всех $1<l<s$ лежат в $\theta_{v(l)}(R / v(l) R)$. При этом в качестве $F_{2}$ можно взять группу, строго изоморфную $F$, определенную в первой части предложения 1.1.1.

Обозначим логарифм $F^{\prime}$ через $\lambda^{\prime}$. Возьмем $\lambda_{s+1}^{\prime}=\lambda^{\prime}(Y)$, где

$$
Y_{i}=X_{i}+\sum_{j} \frac{\theta_{v(s)}\left(f_{i j s}^{\prime}\right)-f_{i j s}^{\prime}}{v(s)} X_{j}^{s}
$$


Согласно определению строгой изоморфности набор рядов $\lambda_{s+1}^{\prime}$ дает логарифм формальной группы над $R$, строго изоморфной $F_{s}$. Возьмем набор рядов $\lambda^{\prime \prime}$, равный "криволинейной части" $\lambda_{s+1}^{\prime}$, т.е. применим первую часть предложения 1.1.1. Из (1) получаем, что для $\lambda^{\prime \prime}$ коэффициенты $f_{i j l}^{\prime \prime}$ при $l<s+1$ принадлежат $\theta_{v(l)}(R / v(l) R)$. Поэтому мы можем взять формальную группу $F_{s+1}$, логарифм которой равен $\lambda^{\prime \prime}$. Предел $F_{s}$ (заметим, что каждый коэффициент стабилизируется, начиная с некоторого места) будет искомой $\theta(F)$, она будет строго изоморфна всем $F_{s}$ (а значит, и $F)$.

Теперь пусть $F$ и $F^{\prime}$ строго изоморфны, различны и удовлетворяют условию на $\theta(F)$ в формулировке теоремы. Пусть $s>1-$ наименьшее число такое, что $F^{\prime} \not \equiv F \bmod \operatorname{deg} s+1$. Тогда $\lambda^{\prime}=\lambda\left(h_{i}\right)$ для некоторых рядов $h_{i} \equiv X_{i} \bmod \operatorname{deg} 2$ при $1 \leqslant i \leqslant m$. Так как $F^{\prime} \equiv F \bmod \operatorname{deg} s$, оба закона криволинейны, мы получаем, что $h_{i} \equiv X_{i}+\sum a_{i j} X_{j}^{s} \bmod \operatorname{deg} s+1$ для некоторых $a_{i j} \in R$. Из формулы (1) мы получаем, что для коэффициентов, при подстановке которых в $F_{e}$ получаются $F$ и $F^{\prime}$, выполнено равенство $f_{i j s}^{\prime}=f_{i j s}+v(s) a_{i j}$. Так как $f_{i j s}$ и $f_{i j s}^{\prime}$ являются представителями элементов $R / v(s) R$, то $a_{i j}=0$ и $F^{\prime} \equiv F \bmod \operatorname{deg} s+1$. Полученное противоречие доказывает утверждение.

2. Доказательство проводится аналогично. Единственное отличие состоит в том, что нужно брать $\lambda_{s+1}$, равный $p$-типической части $\lambda_{s+1}^{\prime}$, т.е. воспользоваться второй частью предложения 1.1.1.

ЗАмЕчаниЕ 2.1.2. Естественный выбор для $\theta=\theta_{p}$ в случае, когда $R$ - кольцо целых полного дискретно нормированного абсолютно неразветвленного поля с совершенным полем вычетов, это система представителей Тейхмюллера. В этом случае представители из пункта 2 нашей теоремы совпадут с каноническими представителями для групп Хонды, построенными в [2] и [3].

ЗАмЕчАниЕ 2.1.3. Из доказательства теоремы видно, что $\theta(F) \bmod \operatorname{deg} r$ зависит только от $F \bmod \operatorname{deg} r$ для каждого $r>1$. Поэтому для групп $F, F^{\prime}$ над $R$ мы имеем $\theta(F) \equiv \theta\left(F^{\prime}\right) \bmod \operatorname{deg} r$, если и только если $F^{\prime}$ строго изоморфна некоторой $F^{\prime \prime}$ такой, что $F \equiv F^{\prime \prime} \bmod \operatorname{deg} r$.

ЗАмечАниЕ 2.1.4. Пусть $A$ - кольцо целых разнохарактеристического локального поля $K, \pi$ - униформизующая $A$. Тогда для формальных $A$-модулей выполнено утверждение, аналогичное пункту 2 теоремы 2.1.1. При этом нужно брать представители $R / \pi R$ в $R$, а вместо $F_{c}$ брать универсальный формальный $A$-типический модуль $F_{V}^{A}$ (см. $\S 21.5$ книги [1]).

2.2. Определенность формальной группы над подкольцом. Пусть $R$ подкольцо в $S$ такое, что для каждого $p \in \mathbb{P}$ мы имеем $R \cap p S=p R$. Получаем, что $R / p R \subset S / p S$. Зададим представители $\theta_{p}$ элементов $S / p S$ так, что $\theta_{p}(R / p R) \subset R$ для каждого $p \in \mathbb{P}$.

ПреДЛОЖЕНИЕ 2.2.1. Группа F строго изоморфна некоторой формалъной группе над $R$ тогда и только тогда, когда $\theta(F)$ определена над $R$.

ДокАЗАтЕЛЬСтво. Утверждение “тогда" очевидно.

Обратное утверждение немедленно следует из того, что для группы $G$ над $R$ существует канонический представитель над $R$, который при наших условиях является также и каноническим представителем над $S$. Поэтому если группа $F$ над $S$ строго изоморфна $G$, то $\theta(F)=\theta(G)$, а значит, формальный групповой закон $\theta(F)$ определен над $R$. 
ЗАмечаниЕ 2.2.2. Если $\mathbb{Z}_{(p)} \subset R$, то мы имеем $R=l R$ для всех простых $l \neq p$, т.е. достаточно проверить, что $R \cap l S=l R$ только для $l=p$. В этом случае, очевидно, предложение 2.2.1 будет выполнено также и для представителей, построенных с помощью $F_{c}$ (т.е. согласно второму пункту теоремы 2.1.1).

\section{3. Поведение классов строгой изоморфности формальных групп при} гомоморфизмах колец. Для кольца $R$ мы обозначим через $i(R)$ множество классов строгой изоморфности формальных групп над $R$. Очевидно, гомоморфизм $f: R \rightarrow S$ индуцирует отображение $f_{i}: i(R) \rightarrow i(S)$.

Докажем основные свойства $f_{i}$.

Теорема 2.3.1. Пусть $R, S$ - кольца без кручения, $f: R \rightarrow S$ - гомоморфизм. Тогда отображение $f_{i}$ инбективно (соответственно сюргективно) тогда и только тогда, когда для каждого $p \in \mathbb{P}$ выполнено $f^{-1}(p S)=p R$ (соответственно выполнено $f(R) \bmod p S=S / p S)$.

ДокАЗАТЕЛЬСтво. 1. Проверка условия инъективности.

Если для каждого $p \in \mathbb{P}$ выполнено $f^{-1}(p S)=p R$, то мы можем выбрать представители $\theta_{p}$ для $S$ и $R$ такие, что представители для $R$ переходят в представители для $S$. Так как $f$ инъективно на представителях для каждого $p$, мы получаем требуемое.

Обратно, пусть существует $p \in \mathbb{P}$ такое, что $f^{-1}(p S) \neq p R$. Выберем такой элемент $z \in R \backslash p R$, что $f(z) \in p S$. Рассмотрим одномерную формальную группу $F_{z}$ с логарифмом, равным

$$
\lambda_{z}=x+\sum_{i>0} \frac{z^{1+p+\cdots+p^{i-1}} x^{p^{i}}}{p^{i}} \in \mathbb{Q} R[[x]] .
$$

Так как $F_{z}$ получается при подстановке в (одномерный) закон $F_{c}$ набора $f_{111}=z$, $f_{11 i}=0$ для $i \geqslant 2$, мы получаем, что $F_{z}$ - формальная группа над $R$. Так как $z / p \notin R$, то $F_{z}$ не может быть строго изоморфна над $R$ аддитивной формальной группе. Так как для каждого $i>0$ мы имеем $f\left(z^{1+p+\cdots+p^{i-1}}\right) / p^{i} \in S$, то $f_{*}\left(F_{z}\right)$ строго изоморфна аддитивной формальной группе над $S$.

2. Проверка условия сюрьективности.

Заменив $R$ на $f(R)$, мы получаем, что достаточно доказывать утверждение в случае, когда $f$ - вложение. Если для каждого $p \in \mathbb{P}$ выполнено $f(R) \bmod p S=S / p S$, то мы можем выбрать представители $\theta_{p}$ для $S$, лежащие в $R$, а значит, каждая группа над $S$ строго изоморфна некоторой формальной группе над $R$.

Обратно, если существует $p \in \mathbb{P}$ такое, что $f(R) \bmod p S \neq S / p S$, то выберем такой элемент $z \in S$, что $z \bmod p S \notin R \bmod p S$. Снова рассмотрим формальную группу $F_{z}$, логарифм которой равен $\lambda_{z}$; она определена над $S$. Проверим, что $F_{z}$ не может быть строго изоморфна формальной группе над $R$. Пусть $F_{z}$ строго изоморфна над $S$ группе $G$ над $R$, ряд $h$ задает строгий изоморфизм. Тогда для эндоморфизмов $[p]$ формального умножения на $p$ мы имееем $[p]_{G}=h \circ[p]_{F_{z}} \circ h^{-1}$, при этом $[p]_{F_{z}} \equiv$ $z x^{p} \bmod (p S, \operatorname{deg} p+1)$. Так как $h \equiv x \bmod \operatorname{deg} 2$, мы получаем, что ряд $[p]_{G}$ также сравним с $z x^{p}$ по модулю $(p S, \operatorname{deg} p+1)$. Таким образом, формальная группа $G$ не может быть определена над $R$. 
ЗАмЕчАниЕ 2.3.2. В частности, мы передоказали результат книги [1] для колец без кручения:

$$
i(R)=i\left(\prod_{p \in \mathbb{P}} \lim _{\longleftarrow} R / p^{s} R\right)=\prod_{p \in \mathbb{P}} i\left(\lim _{\longleftarrow} R / p^{s} R\right) .
$$

ЗАмечАниЕ 2.3.3. Конечно же, если $\mathbb{Z}_{(p)} \subset R$, то достаточно проверять условия теоремы для единственного простого числа, равного $p$.

Автор благодарен С. В. Востокову за полезные замечания.

\section{СПИСОК ЦИТИРОВАННОЙ ЛИТЕРАТУРЫ}

[1] M. Hazewinkel, Formal Groups and Applications, Pure and Applied Mathematics, 78, Academic Press, New York-London, 1978.

[2] М. В. Бондарко, С. В. Востоков, “Явная классификация формальных групп над локальными полями”, Теория чисел, алгебра и алгебраическая геометрия, К 80-летию со дня рожд. акад. И. Р. Шафаревича, Тр. МИАН, 241, № 2, Наука, М., 2003, 43-67.

[3] М. В. Бондарко, "Явная классификация формальных групп над полными дискретно нормированными полями с несовершенным полем вычетов", Тр. СПб МО, 11, 2005, $1-36$.

[4] T. Honda, "On the theory of commutative formal groups", J. Math. Soc. Japan, 22 (1970), 213-246.

М. В. Бондарко

Санкт-Петербургский государственный университет
Поступило

04.02.2004

Исправленный вариант

04.12 .2006 